\title{
Real-Time Modeling of Transverse Emittance Growth Due to Ground Motion
}

\author{
V. D. Shiltsev and V. V. Parkhomchuk \\ Superconducting Super Collider Laboratory* \\ 2550 Beckleymeade Ave. \\ Dallas, TX 75237 USA \\ and \\ Budker Institute of Nuclear Physics \\ Novosibirsk, Russia
}

September 1993

*Operated by the Universities Research Association, Inc., for the U.S. Department of Energy under Contract No. DE-AC35-89ER4i486.

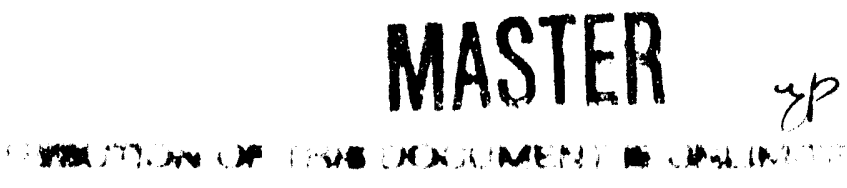




\title{
Real-Time Modeling of Transverse Emittance Growth Due to Ground Motion
}

\author{
V. D. Shiltsev and V. V. Parkhomchuk
}

\begin{abstract}
Ground motion noise at frequencies around $1 \mathrm{kHz}$ causes growth of transverse emittance of the Superconducting Super Collider (SSC) collider beams. The effect was quantitatively investigated using real-time signals from seismometers installed at the tunnel depth and on the surface. The SSC beam was modeled as an ensemble of oscillators with a spread of betatron frequencies. The effect of transverse feedback on emittance growth was investigated.
\end{abstract}




\subsection{INTRODUCTION}

Vibrations of magnetic elements of the Superconducting Super Collider (SSC) can seriously disturb proper machine operation. At first, such vibrations cause distortions of the closed orbit. From this point of view ground motion at frequencies below $20 \mathrm{~Hz}$ is dangerous because of large amplitudes. On the other hand, the revolution frequency of the SSC, $f_{0}=3.44 \mathrm{kHz}$, will be the smallest arnong particle accelerators, and vibrations at frequencies of approximately $1 \mathrm{kHz}$ and above will lead to direct emittance growth. Careful analytical investigation of the last effect, reported in a series of articles, ${ }^{1-3}$ has shown that emittance growth is proportional to the value of the power spectral density (PSD) of vibrations at resonance frequencies $f_{0}|v-n|$, where vis the tune and $n$ is an integer. These oscillations resonantly drive the betatron oscillations of the beam. Due to decoherence, these oscillations rapidly translate into growing emittance of the beam. We will discuss only emittance growth issues.

Previous measurements of ground vibrations ${ }^{4,5}$ have shown that the PSD at the resonant frequencies is less than the allowable level of $3^{*} 10^{-12} \mu \mathrm{m}^{2} / \mathrm{Hz}^{1,2}$ only under quiet conditions. On weekdays and in the presence of nearby technological activities, the vibrations can be far above this value. The transverse feedback system was suggested as the most effective way to keep emittance growth at an acceptable level. $1,2,6$

In this paper we present the results of real-time modeling of emittance growth due to ground motion at the SSC collider. In this model the beams were represented as ensembles of oscillators on which the online measured signals of ground motion act for up to $24 \mathrm{~h}$ (approximately the cycle time of the collider). The effects of tune choice and of transverse feedback are considered.

In Section 2.0, a description of the computer model is given, together with an explanation of the interaction of the software program with seismoprobe signals. Section 3.0 is devoted to the principal results of the modeling described. In Section 4.0 we present brief conclusions and a discussion of measurements.

\subsection{DESCRIPTION OF MEASUREMENTS AND BASIC COMPUTER MODEL}

To excite the computer beams we took on-line signals from two seismometers that were used for measurements of vertical ground vibrations at the exploratory shaft of the SSC. The seismometers are SM-3KV type velocity meters. The meter's quantitative characteristics are described in detail in Reference 5. The probes served for measurements of vibrations in the frequency band of $0.05 \mathrm{~Hz}$ to $1.5 \mathrm{kHz}$. One of the probes was insta!led in the horizontal adit of the exploratory shaft at a depth of $66 \mathrm{~m}$, and the other was set at the surface. Hereafter they will be referred to as "down" and "top," respectively. Signals from these probes were digitized simultaneously by ADCs with a sampling frequency of $3.44 \mathrm{kHz}$ (equal to the revolution frequency of the collider) and then were sent to an IBM 386 computer through a CAMAC-PC interface. All developed computer programs were integrated in the ASSA ${ }^{5}$ seismic station software package, and data was stored in CAMAC memory or in PC files. While probes were precalibrated (see data in Reference 5), the signals were easily transformed in units of displacement and used for calculation of the vertical kick of $20-\mathrm{TeV}$ protons due to the same displacement of the SSC quadrupole. For example, the sensitivity of SM-3KV probes at frequencies of $700 \mathrm{~Hz}-95 \mathrm{r} \mathrm{Hz}$ is approximately $9.2 \mathrm{~V} / \mu \mathrm{m}$.

Our recent and previous measurements 5,7 have shown that at frequencies above $100 \mathrm{~Hz}$, vibrations of the ground at two points $90 \mathrm{~m}$ apart-the distance between neighboring quadrupoles in the collider-are uncorrelated within the accuracy of measurements. This allowed us to further simplify beam dynamics modeling and to replace the influence of $N \approx 1000$ kicks from all quadrupoles around the ring by an effective one-turn kick with amplitude $\sqrt{N} \approx 31.6$ times larger.

Let us consider the main features of the computer model we used. The model solved for twodimensional phase-space motion of an ensemble of particles (up to 1000) in a beam. The motion of each 
particle has been modeled as a sequence of mappings governing evolution of the phase-space coordinates $x$ and $p$ :

$$
x=\frac{X}{\sqrt{\beta}}, p=\beta \frac{d X}{d s \sqrt{\beta}},
$$

where $X$ stands for the particle deviation with respect to the closed orbit, $\beta$ is the beta function, and $s$ is the path length along the orbit. First, the following one-turn linear map described the particle motion in the lattice:

$$
\left(\begin{array}{l}
x_{j}^{\prime} \\
p_{j}^{\prime}
\end{array}\right)=\left(\begin{array}{cc}
\cos 2 \pi v_{j} & \sin 2 \pi v_{j} \\
-\sin 2 \pi v_{j} & \cos 2 \pi v_{j}
\end{array}\right)\left(\begin{array}{l}
x_{j} \\
p_{j}
\end{array}\right),
$$

where $j=1,2, \ldots, M$ numerates particles, and $v_{j}$ is the tune of thej-th particle. During modeling, tunes of beam particles were distributed according to the Gaussian law with an rms value of 0.0003 (which describes well the decoherence process due to beam-beam interactions with the parameter $\xi=0.0018$ (Reference 8 )) and with the variable mean value. The effects of ring non-linearity and synchrotron motion were neglected in our simulations.

The effect of kicks in all of the ring magnets due to ground motion on one turn was modeled by the transformation:

$$
p_{j}^{\prime}=p_{j}+\frac{\Delta}{\sqrt{2}}, x_{j}^{\prime}=x_{j}+\frac{\Delta}{\sqrt{2}}
$$

where $\Delta$ characterizes the effective one-turn kick taken from the seismoprobe. The sum of the kicks yields equal increments for coordinates and momenta because of the independence of kicks from different magnets and betatron oscillations.

The presence of the feedback system was simulated in accordance with

$$
p_{j}^{\prime}=p_{j}-g \bar{p}, \bar{p}=\frac{1}{M} \sum_{j=1}^{M} p_{j}
$$

As one can see from Eqs. (3) and (4), all particles experience the same kick at the noise and feedback transformations.

Emittance of the beam has been computed as

$$
\varepsilon=\frac{1}{M} \sum_{j=1}^{M}\left(\left(x_{j}-\bar{x}\right)^{2}+\left(p_{j}-\bar{p}\right)^{2}\right) .
$$

This definition of emittance reflects only sizes of the beam and avoids variations of the emittance related to coherent beam motion. 
For modeling of emittance growth in the presence of a feedback system, we chose the parameters of gain $g$ and number of particles $M$ in order to make decrements of artificial stochastic cooling much smaller than the observed emittance growth rate. (See, for example, Reference 1.)

\subsection{RESULTS OF MODELING}

Results of emittance growth simulations during a 20-s time period are shown in Figure 1(a). Figure 1(b) presents input data from the seismometer placed at the tunnel depth. The emittance growth modeling was done with a mean tune of beam of 123.265 , or its fractional part of 0.265 ; i.e., the first resonance frequency that led to emittance growth is $0.265 * 3443 \mathrm{~Hz}=912 \mathrm{~Hz}$. Figure 1 shows that normalized emittance grows practically linearly in time. Note that the design emittance of the SSC beams is $1 \mathrm{~mm}^{*} \mathrm{mrad}$. The data presented were measured at $15: 36$, July 14,1993 , under comparably quiet conditions.

(a)

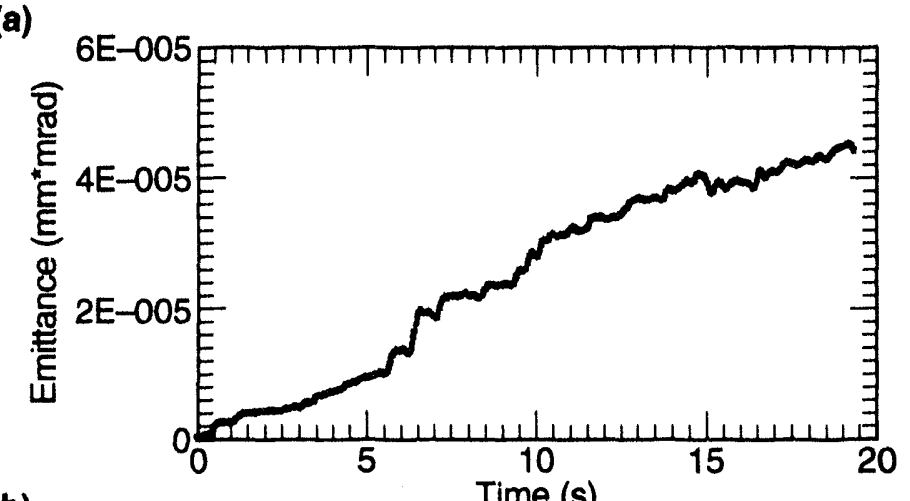

(b)

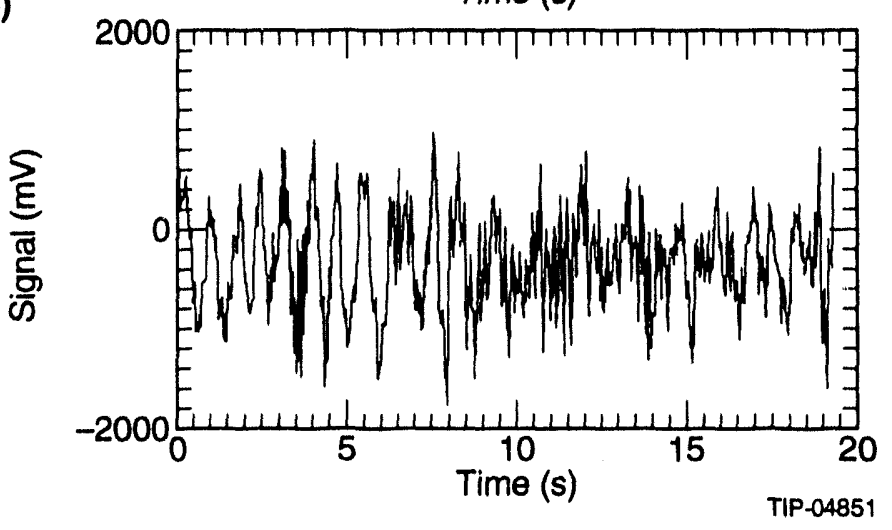

Figure 1. (a) Increment of Normallzed Transverse Emittance Corresponding to Probe Signal; (b) Signal from SM-3KV Probe Installed at the Tunnel Depth.

Figure 2 presents emittance growth modeling with the same beam parameters, but under conditions when the experimenter struck the ground with a $2-\mathrm{kg}$ hammer about $15 \mathrm{~m}$ from the "top" seismometer, which was used as source of ground motion signal for the computer's beam. Figure 2 (a) presents input signal from the gauge in time domain; Figure 2(b) the emittance growth without feedback system; and Figure 2(c) the simultaneously calculated emittance growth when the feedback system with $g=0.05$ was used. Step behavior of emittance increments is clearly seen. One can see that the feedback system allows a 250-fold decrease in emittance growth. 
(a)

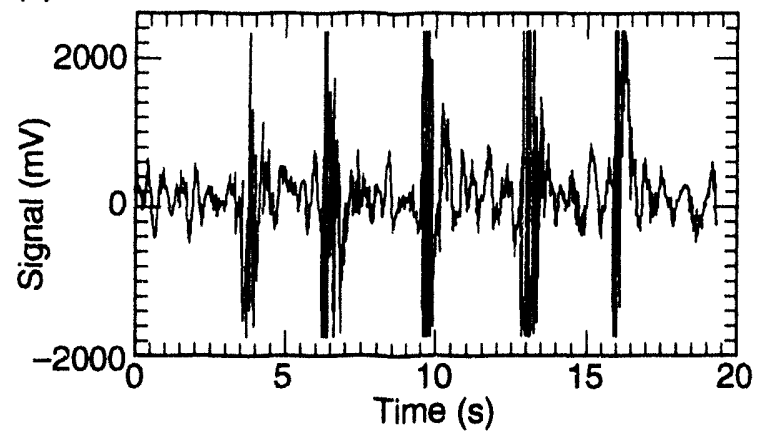

(b)

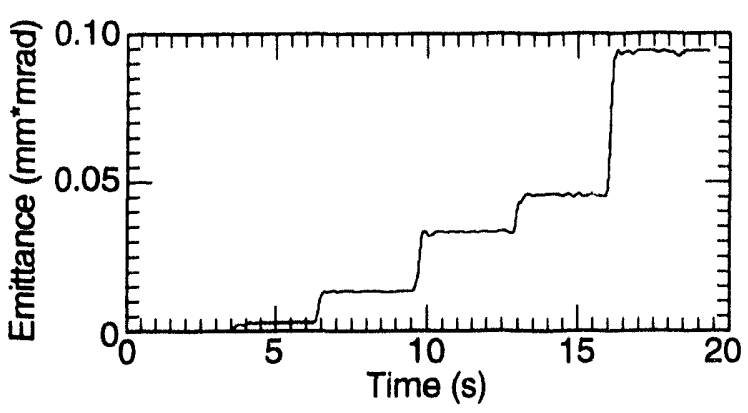

(c)

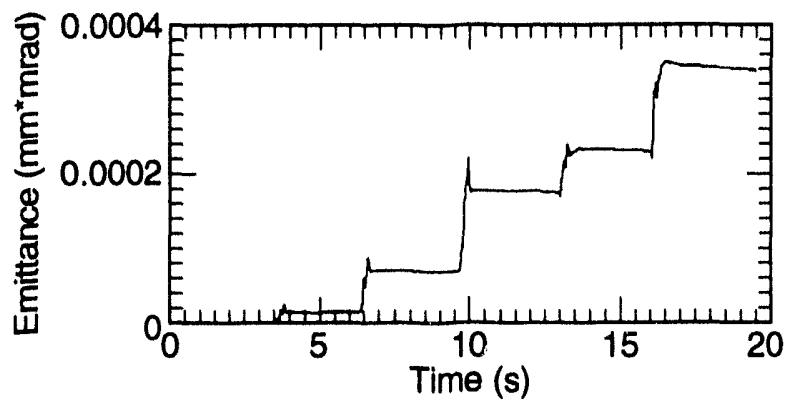

TIP-04852

Figure 2. (a) Signal from "Top" Selsmoprobe During a Few Strlkes by a Hammer 15 m Away;

(b) Corresponding Increment of Transverse Emittance;

(c) Increment of Emittance with Feedback System with Gain $g=0.05$.

Results of long-term (24 h from 20:00, Saturday, August 28, until 20:00, Sunday, August 29) observations of emittance growth in quiet conditions are presented in Figure 3. The fractional part of the tune was equal to 0.265 , as in previous examples. Curves 1 and 2 correspond to the "top shaft" and "down shaft" conditions. Curve 3 presents the growth of emittance under the influence of noise from seismoprobes, amplifiers, and ADCs (probe was rigidly fixed); i.e., it speaks to the accuracy of our predictions. One can see that at a frequency of approximately $900 \mathrm{~Hz}$, signal-to-noise ratio was about 6 , even for the low-signal case. Log-log scale cf Figure 3 also shows that, in general, emittance grows linearly in time if one disregards minor details. While the increment of emittance is about $60 \%$ (even without taking into consideration synchrotron radiation damping), one can see that such an environment is not dangerous for the SSC beams.

Another example of emittance growth due to ground vibrations at the tunnel depth during a working day (from 23:00, August 30, until 19:00, August 31, 1993) is shown in Figure 4. There were the usual weekday conditions, with heavy machinery working around the SSC Linac site. Figure 4(a) presents emittance growth without feedback (see curve 1) and including in the computation 20-h damping time for betatron oscillations due to synchrotron radiation of protons in the SSC rings (see curve 2). One can see that the effect of cultural noise leads to as much as a 30-fold increase in emittance over the design value for normalized emittance. Simultaneous modeling of beam dynamics with a feedback system with $g=0.05$ shows that the systems allow a 150 -fold decrease in emittance, down to an acceptable level of $20 \%$. (See Figure 4(b).) The fractional part of the tune in this modeling was equal to 0.14 . 


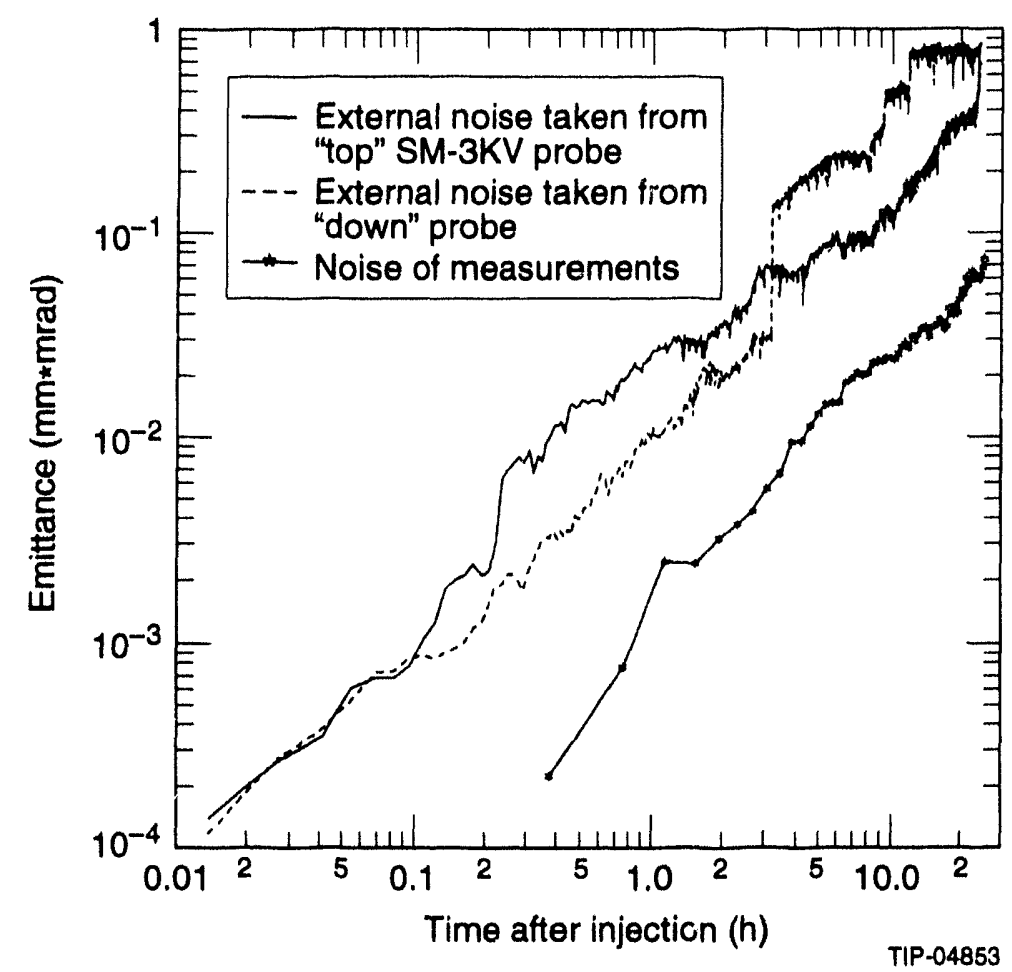

Figure 3. Results of 24-h On-line Simulations of Emittance Growth Under Quiet Conditions.

(a)

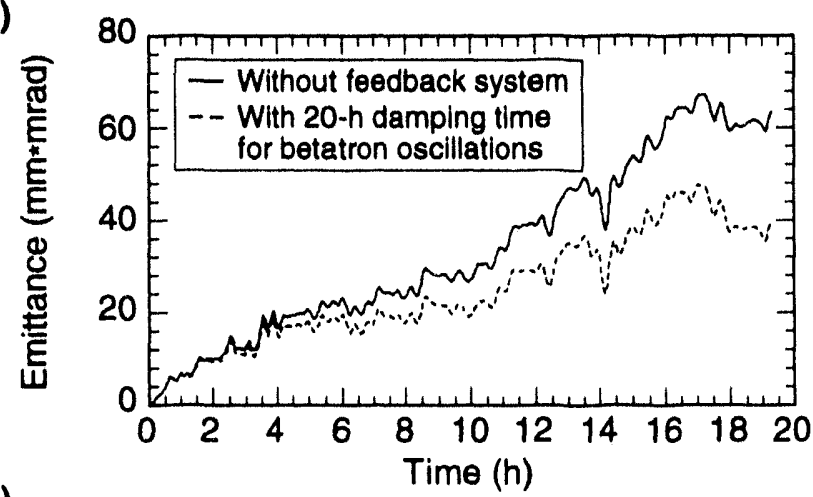

(b)

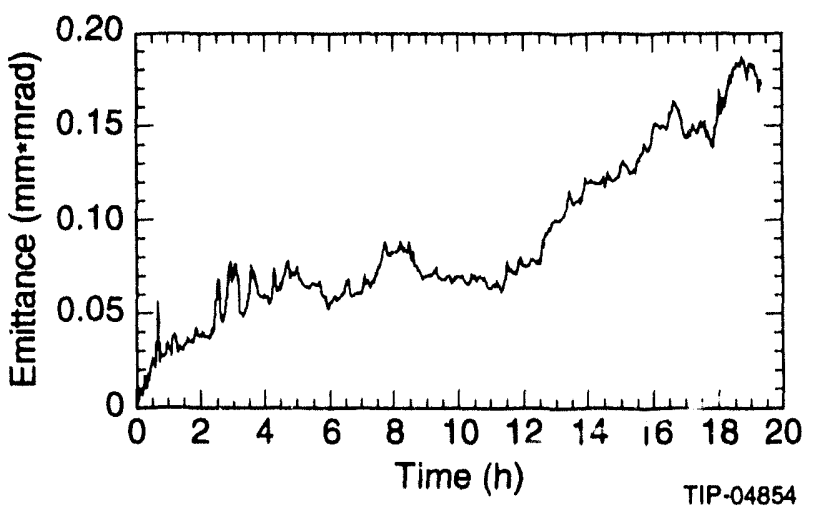

Figure 4. 20-h On-ilne Simulations of Emittance Growth During Nolsy Weekday;

(a) Without Feedback System and with 20-h Damping Time;

(b) With Feedback System with $g=0.05$. 


\subsection{DISCUSSION AND CONCLUSIONS}

Let us compare the effect of the feedback system on emittance growth with the theoretical investigation of the issue made in References 1 and 2 . In these articles it was shown that the feedback system with gain $g$ will decrease the emittance growth by a factor of $R$ :

$$
R=\left(\frac{g}{4 \pi \Delta v_{r m s}}\right)^{2}
$$

where $\Delta v_{r m s}$ is the rms tune spread. With parameters of our simulations $\Delta v_{r m s}=0.0003$ andg $=0.05$, $R=176$, which is within $30 \%$ of the value of the simulations.

Another interesting test in our theoretical investigations was applied to the formulas (19) in Reference 2. These formulas are concerned with emittance growth in an array of oscillators through the PSD $S(f)$ of external noise, which acts on the coherent motion of oscillators by periodic (with frequency $\left.f_{0}\right) \delta$-functional kicks:

$$
\frac{\Delta \varepsilon}{\Delta t}=\frac{f_{0}^{2}}{2} \Sigma(v), \Sigma(v)=\sum_{n=-\infty}^{\infty} S\left(f_{0}(v-n)\right)
$$

We used two methods to make a spectral analysis of the same data array that presented a signal from one of the velocity meters. The first method was to calculate the emittance growth rate with different fractional part of the tune $v$, and then find $S(f)$ from Eq. (7) at the first resonant frequency (the PSD falls quickly as $f$ increases). The second method was the usual harmonic analysis. Figure 5 presents a comparison of PSD of the SM-3KV signal found with use of Eq. (7) while sweeping $v$ and with the help of the Fast Fourier Transformation program with Antimed digital filter. ${ }^{5}$ One can see a good coincidence of the two ways, i.e., an adequacy of the language of PSD for problems of emittance growth.

Let us list several conclusions of our work:

1. Measurements of ground motion in an underground experiment in the exploratory shaft of the SSC allowed us to simulate emittance growth directly in an on-line regime. In quiet conditions (absence of man-made noises), the rate of emittance growth was found to be a factor of about 3 below the acceptable level of $1 \mathrm{~mm} * \mathrm{mrad} / 20 \mathrm{~h}$.

2. We found that cultural (technical) seismic noise can cause unacceptably fast growth of transverse emittance (about 30 times the $1 \mathrm{~mm}^{*}$ mrad level during $24 \mathrm{~h}$ of beam lifetime).

3. There are few ways to solve the issue: a) to decrease these noises to a minimum; b) to increase the value of the fractional part of the tune, because in this case the beam will be sensitive to higher frequencies, which usually have smaller amplitudes; c) to install a transverse feedback system to damp coherent betatron oscillations.

4. In our simulations the feedback system strongly damped the emittance growth rate below the acceptable level. 


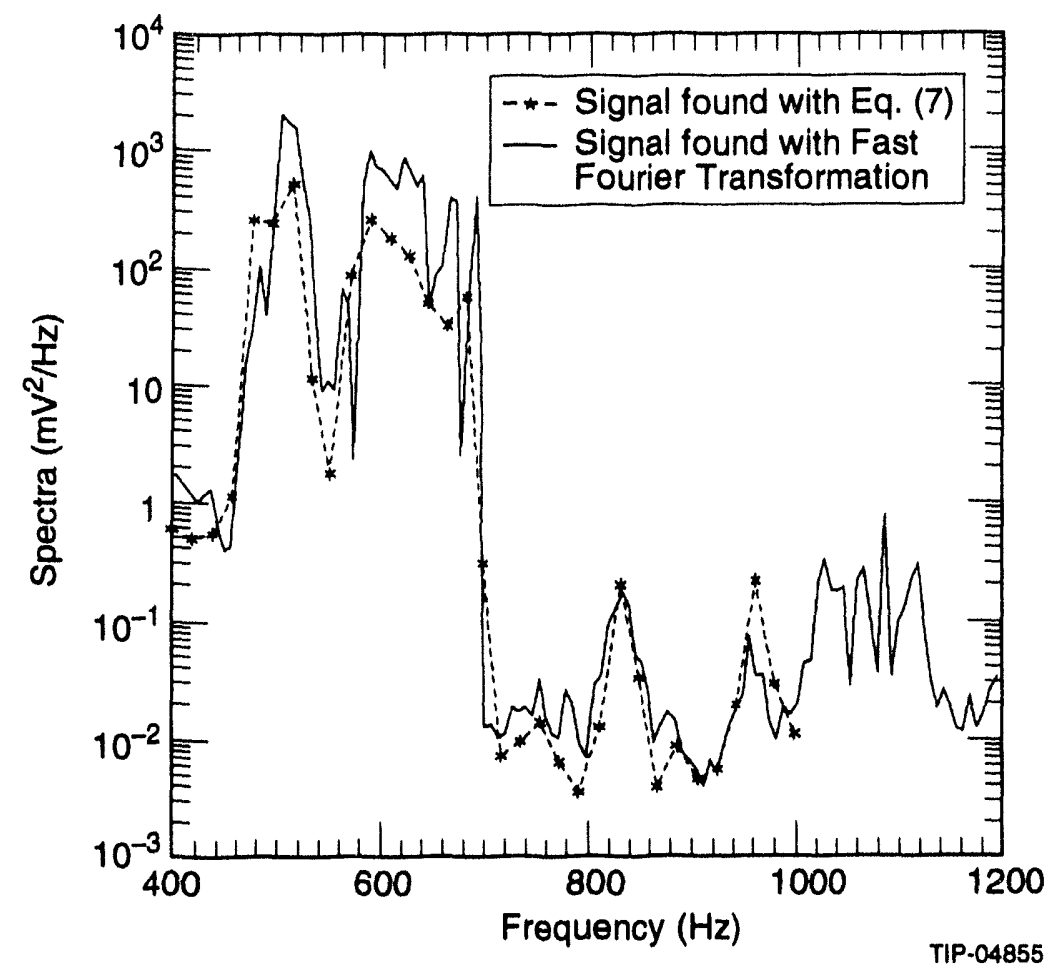

Figure 5. Comparison of Two Different Ways to Calculate PSD of Signal. (See comments in text.)

\section{ACKNOWLEDGMENTS}

The authors are thankful to G. Stupakov, R. Meinke, V. Lebedev, and E. Tsyganov for their interest in this work.

We acknowledge Gennady Stupakov for careful reading and commenting the manuscript. 


\section{REFERENCES}

1. V. A. Lebedev, V. V. Parkhomchuk, V. D. Shiltsev, and A. N. Skrinsky, "Suppression of Emittance Growth Caused by Mechanical Vibrations of Magnetic Elements in the Presence of Beam-Beam Effects in the SSC," INP Preprint 91-120, Novosibirsk, 1991.

2. V. Lebedev, V. Parkhomchuk, V. Shiltsev, and G. Stupakov, "Emittance Growth Due to Noise and Its Suppression with the Feedback System in Large Hadron Colliders," SSCL-Preprint-188, March 1993.

3. G. V. Stupakov, "Emittance Growth Caused by Magnet Vibrations in the SSC," Preprint SSCL-516, March 1992.

4. V. Parkhomchuk, V. D. Shiltsev, and H. J. Weaver, "Measurements of the Ground Motion Vibrations at the SSC," SSCL-Preprint-323, May 1993; and Proc. of 1993 IEEE Part. Acc. Conf., May 1993, Washington, DC.

5. V. V. Parkhomchuk, V. D. Shiltsev, and H. J. Weaver, "Measurements of Ground Motion and SSC Dipole Vibrations," SSCL-624, June 1993.

6. V. V. Parkhomchuk and V. D. Shiltsev, "Is Transverse Feedback Necessary for the SSC Emittance Operation? (Vibration Noise Analysis and Feedback Parameters Optimization)," SSCL-622, June 1993.

7. V. D. Shiltsev, V. V. Parkhomchuk, and H. J.Weaver, report on underground seismic measurements in the SSC exploratory shaft, to be published.

8. G. V. Stupakov, V. V. Parkhomchuk, and V. D. Shiltsev, "Decoherence of a Gaussian Beam Due to Beam-Beam Interaction," SSCL-Preprint-495, 1993. 

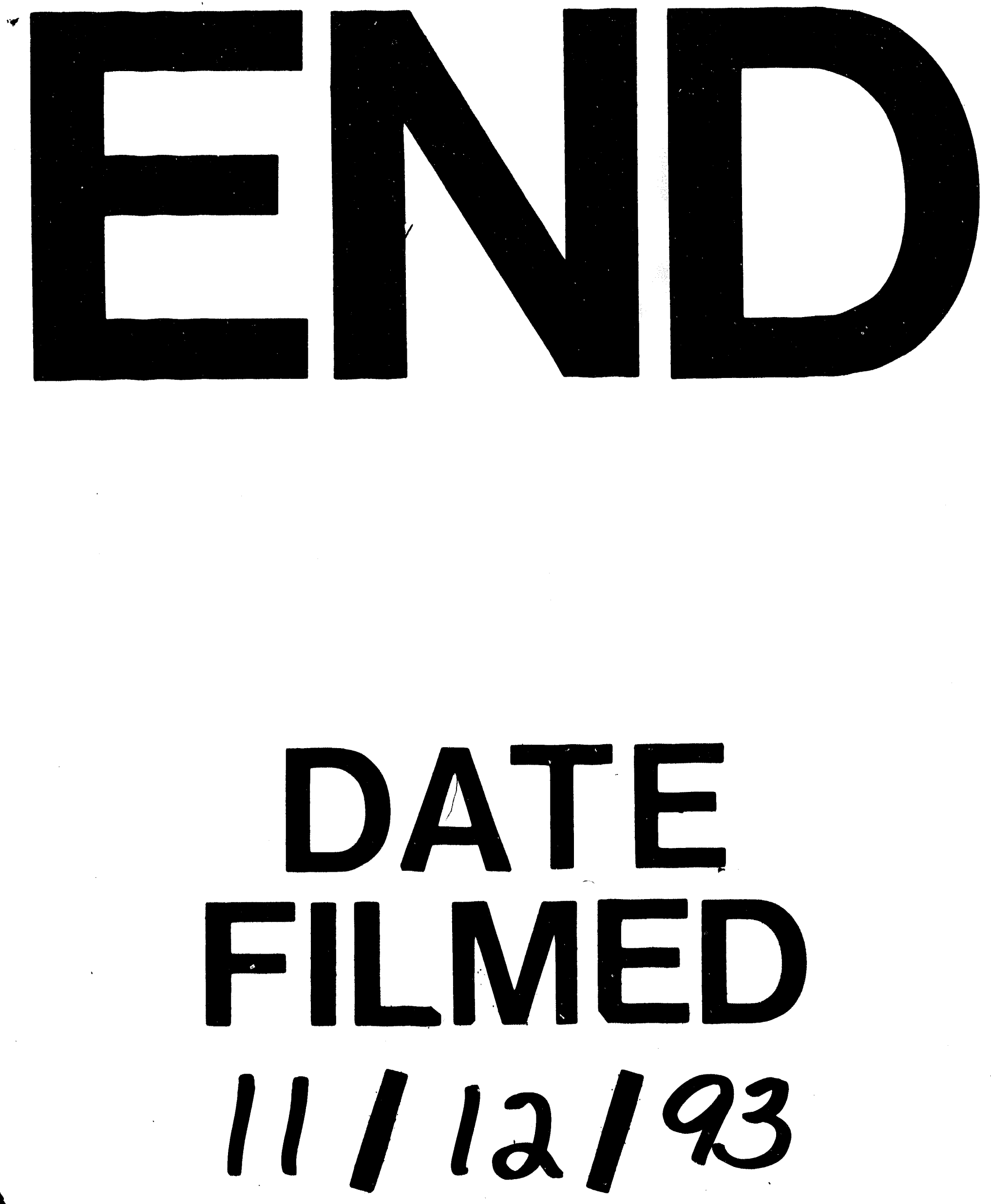

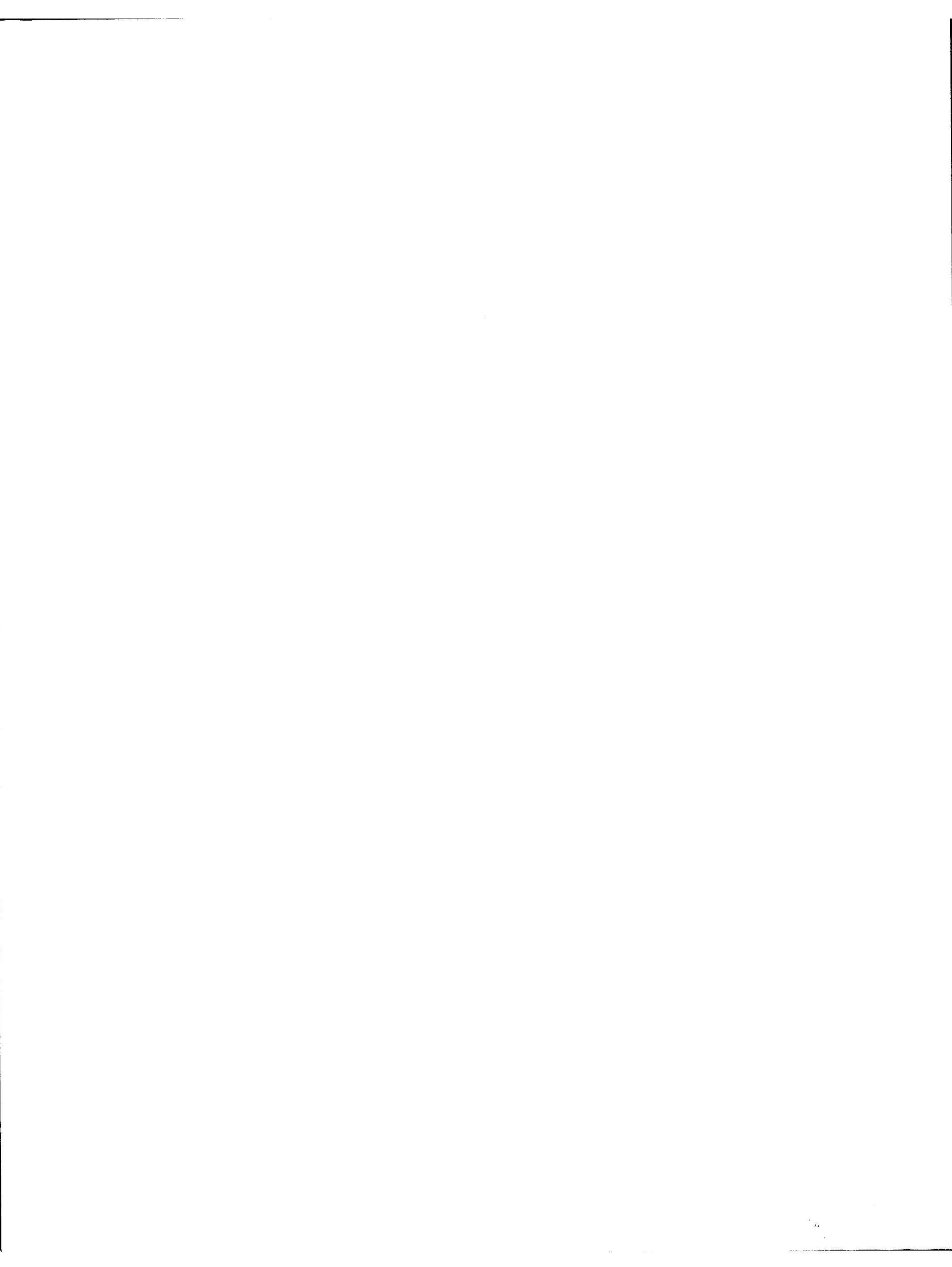
$-$ 